ERRATUM

\title{
IMPLICATIONS OF A LARGE Htit-COUPLING FOR THREE-BODY DECAYS OF HIGGS BOSON AND TOP QUARK
}

[Mod. Phys. Lett. A, Vol. 6, No. 38 (1991) 3491-3497]

R. DECKER, M. NOWAKOWSKI and A. PILAFTSIS

\begin{abstract}
Note that in Fig. 1 the branching ratio for the decay channel $H^{0} \rightarrow t \bar{t} Z^{0}$ has to be multiplied by the color factor 3 , which, unfortunately, has not been taken into account in our numerical considerations. In any case, an extensive and complete analysis of all three-body decays of the Higgs and top quark will be presented by us in the near future.
\end{abstract}

